\title{
The Relationship between Medical Students' Family Cohesion, Adaptation and Learning Burnout: the Mediating Effect of Psychological Resilience
}

\author{
Yongmei Hou ${ }^{a}$, Riman Liang
}

\author{
Department of Psychology, School of Humanities and Management, Guangdong Medical \\ University, Guangdong \\ a352314640@qq.com
}

Keywords: Learning Burnout, Family Cohesion, Family Adaptability, Resilience, Mediating effect.

\begin{abstract}
Objective To investigate the characteristics of resilience family cohesion, adaptability and learning burnout, and analyse the relationship among them. Methods: One thousand six hundred and twenty- five undergraduates who were collected from Seven Universities in Guangdong by stratified random sampling method were investigated with Learning Burnout Scale for Chinese Undergraduates (LBSCU), Family Adapation and Cohension Scale, Second Edition-Chinese Version (FACESII-CV) and Resilience Scale for Chinese Adolescent (RSCA). Result: (1) The total score of LBSCU was in the medium level. (2) There was a significantly positive correlation to each other among the total score of LBSCU, the total score of RSCA, the score of family Cohension and family adapation $(\mathrm{r}=-0.179 \sim-0.388$, Ps $<0.05)$. (2) It had a partially mediating effect of the total score of RSCA when the score of family Cohension was taken as a predictor to the total score of LBSCU, and the ratio was 55.8\%. (3)It had a partially mediating effect of the total score of RSCA when the score of family adaption was taken as a predictor to the total score of LBSCU, and the ratio was 65.3\%. Conclusion: Both family Cohension and family adapation had a significant effect on the views on marriage and love by resilience in undergraduates.
\end{abstract}

\section{Introduction}

Learning burnout comes from researches about occupational burnout and till now, there is still not a consistent definition about it. Pines thinks that "learning burnout" is "a kind of phenomenon in which students suffer from energy exhaustion, passion loss toward their studying and school activities, and emotional indifference and alienation toward their schoolmates due to long-term learning load and worse-than-expected learning performance, ${ }^{\text {,1] }}$. Lian Rong ${ }^{[2]}$ thinks that learning burnout is "the negative attitude and behavior of students' tiredness about their studying due to studying pressure or lack of studying interest.” Although the descriptions are different, scholars all think that the core problem of learning burnout is "being inactive about learning".

Learning burnout is the most common problem appearing among undergraduates, which detection rate abroad is $9.9-40.3 \%^{[3-4]}$, domestically being $24.8-51.3 \%^{[5-6]}$. It can reduce individual learning initiative ${ }^{[7]}$, leading to physical and mental fatigue[8] and psychological syndrome ${ }^{[9-10]}$, and hindering learning and occupational development ${ }^{[11-12]}$.

Psychological resilience is the excellent adaptive ability(process) individuals present when facing adversities or tremendous pressure, it is a process through a series of dynamic interactions among abilities and qualities, which can make individuals rapidly restore and successfully take countermeasures when suffering from significant pressure and danger ${ }^{[13]}$.

Burnout happens with the reduction of life or work satisfaction. Satisfaction reduction means 
individuals are faced with difficulties. If they can't handle successfully, burnout happens consequently ${ }^{[14-15]}$. Psychological resilience refers to the competences and psychological qualities individuals need to cope with difficulties and realize rapid restoration ${ }^{[13]}$. Thus, psychological resilience is a psychological quality to cope with learning difficulties while learning burnout is the outcome when individuals can't cope with their learning difficulties ${ }^{[15]}$.

Family is the basis individuals rely on to live, which basic function is to provide necessary environment and conditions for the healthy development of family members in physical, mental, social and other aspects. Family not only directly influences various aspects of children's socialization $^{[16]}$, but also has a paramount effect on individuals' mental health ${ }^{[17-18]}$. As the important content of psychological qualities and a common psychological problem of undergraduates respectively, psychological resilience and learning burnout should be subject to the influence of family functions, which are mainly reflected in two big aspects, namely, family cohesion and adaptation. The former means the emotional connection among family members, while the later refers to the ability that family system can make corresponding changes for the problems appearing in its different development stages ${ }^{[19]}$. We can infer that, family cohesion and adaptation not only directly influence the creation of learning burnout, but also directly influence the formation of psychological resilience. Further, they can influence the creation of learning burnout indirectly through psychological resilience.

On the other hand, from the empirical perspective, family functions, psychological resilience and learning burnout have close relationship. Psychological resilience has a significant negative correlation with learning burnout ${ }^{[20-21]}$, family cohesion and adaptation both have significant positive correlations with psychological resilience ${ }^{[22]}$, while family environment is a predictive variable of learning burnout ${ }^{[23-24]}$. We can hypothesize that psychological resilience creates a mediating effect among family cohesion, adaptation and learning burnout. The mediating effect models are shown in Figure 1, 2 and 3 as below.
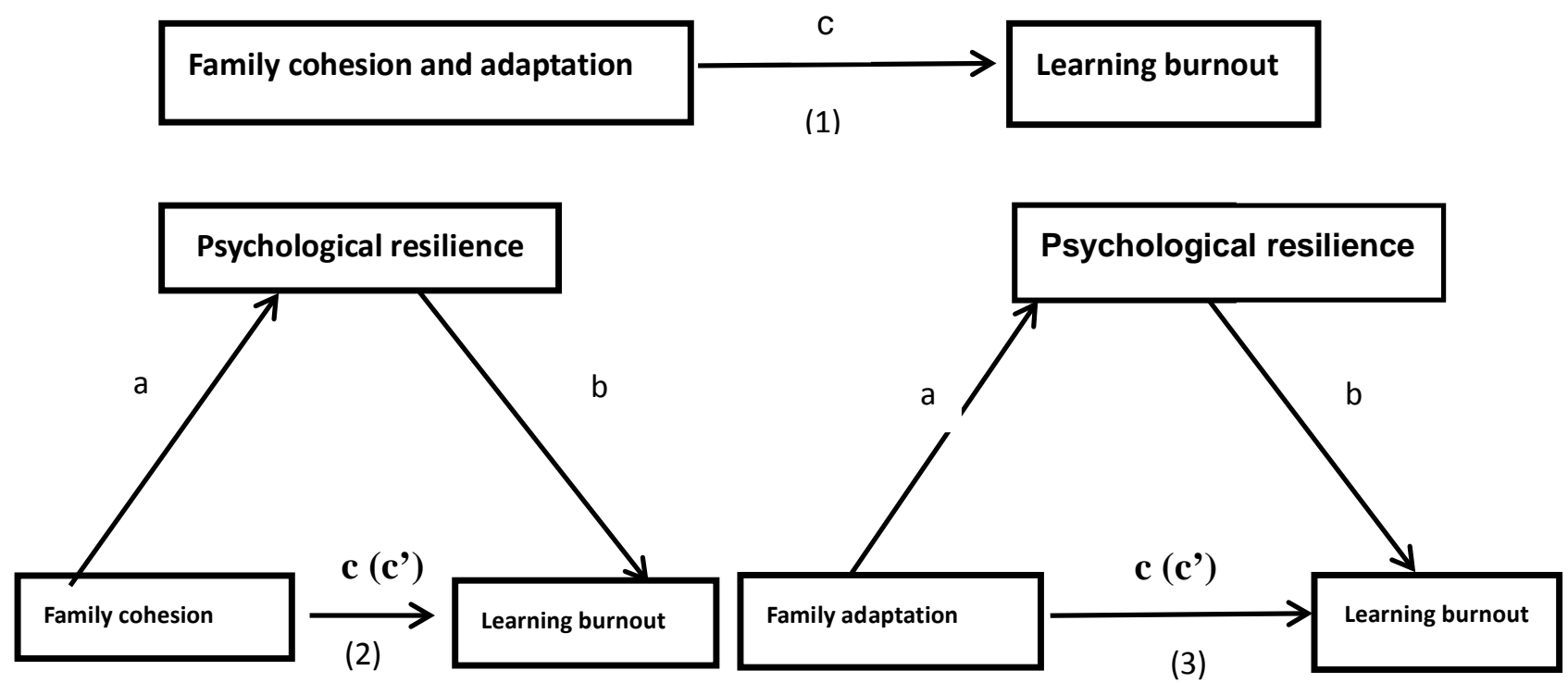

\section{Attention:}

(1)Figure 1: the predictive effect of family cohesion and adaptation on learning burnout;

(2)Figure 2: the mediating effect of psychological resilience on family cohesion and learning burnout;

(3)Figure 3: the mediating effect of psychological resilience on family adaptation and learning burnout. 


\section{Object and Method}

\subsection{Object}

The stratified randomization is adopted to select 1750 undergraduates majoring in clinical medicine from 7 medical colleges of Guangdong province, in which 50 medical students are selected randomly from every grade of every school. 1625 valid questionnaires were reclaimed, resulting in an effective rate of 92.9\%. Among all the respondents, the number of male students is 687(39.8\%), female ones 938(60.2\%), freshmen 421(25.9\%), sophomores 389(23.9\%), juniors 405(24.9\%), seniors 317(19.5\%), grade five students 93(5.7\%).

\subsection{Research Tools}

\subsubsection{Learning Burnout Scale for Chinese Undergraduates, BBSCU $^{[2]}$}

This scale is formulated by Lian Rong, including 20 items and 3 factors, that is, down spirit, improper conduct and low sense of achievement. It adopts Likert 5-grade rating system, 1 to 5 points corresponding to the respective degrees from "completely non-conformant" to "completely conformant". The higher the point is, the higher the degree of learning burnout is. In this research, the reliability of this scale re-tested after one month is 0.822 , the correlation coefficient among various factors and the full scale is $0.771-0.890(\mathrm{P}<0.001)$, the Cronbach $\alpha$ coefficient of the full scale is 0.864 while that of various factors is $0.733-0.814$.

2.2.2 Family Adapation and Cohension Scale, Second Edition-Chinese Version, FACESII-CV ${ }^{[25]}$

Formulated by Olson, translated by Shen Qijie and etc., and revised by Zhou Dinghui and etc., this scale is a self-evaluation one, including 30 items and 2 component scales. It is mainly used to evaluate family functions in the following 2 aspects: 1)cohesion: refers to the intimacy degree of emotional connection among family members; (2)adaptation: refers to the ability that the family system makes corresponding changes to the problems appearing in different family conditions and different family development stages. It adopts Likert 5-grade rating system, 1 to 5 points corresponding to the respective degrees from "not be" to "always be", the answers of correspondents represent the degree in which the status depicted by the item occurs in the family. In this research, the reliability of this scale re-tested after one month is 0.848 , the correlation coefficient among various component scales and the full scale is $0.742-0.874(\mathrm{P}<0.001)$, the Cronbach $\alpha$ coefficient of the full scale is 0.895 while that of various component scales is 0.758-0.906.

2.2.3 Resilience Scale for Chinese Adolescent, RSCA) ${ }^{[26]}$

This scale is formulated by Gan Yiqun and Hu Yueqin, including 27 items and 5 dimensions, that is, target focus, positive cognition, emotion control, interpersonal help and family support. It adopts Likert 5-grade rating system covering respective degrees from "completely non-conformant" to "completely conformant", in which "completely non-conformance" is marked with 1 point, “comparably non-conformant” 2 points, "uncertain” 3 points, "comparably conformant” 4 points, "very conformant" 5 points. The higher the point is, the more apparent the inclination in the item(dimension) is(Hu Yueqin, Gan Yiqun, 2008). In this research, the reliability of this scale re-tested after one month is 0.841 , the correlation coefficient among various dimensions and the full scale is $0.693-0.829(\mathrm{P}<0.001)$, the Cronbach $\alpha$ coefficient of the full scale is 0.833 while that of various dimensions is $0.736-0.883$.

\subsection{Statistical Analysis}

This research adopts spss.16.0 software package to process the data while utilizing descriptive statistics, independent sample t test, one-way variance analysis, linear regression analysis for its statistical analysis. 


\section{Results}

\subsection{Data decentration}

In order to avoid the influence of multicollinearity among various variables, based on the definition of mediating variables, data with corresponding dimensions are decentralized.

\subsection{Statistic description}

Table 1 gives the mean value, standard deviation and correlation coefficient of various research variables. It can be inferred from this table that the total score of learning burnout scales falls in the medium range. Within 3 dimensions, improper conduct gets the highest mean score while low sense of achievement has the lowest. According to Olson ${ }^{[27]}$,s family "Circumpolar Model"(Olson and etc., 1983), there are 16 family types based on their different family cohesion and adaptation. Combined with the data in table 6, the family type of medical students mostly belongs to "regular-intimate(balanced type)".

The results from relevant analysis indicate that the correlation between every two of medical students' total score of learning burnout scales, family cohesion, family adaptation, total score of psychological resilience scales all reaches a remarkable level $(\mathrm{P}<0.05)$, it is worthy to further check the mediating effect of psychological resilience between family cohesion and learning burnout, family adaptation and learning burnout.

Table 1 The descriptive statistics of various research variables and correlation analysis results $(\mathrm{n}=1625)$

\begin{tabular}{|c|c|c|c|c|c|c|c|c|c|c|c|c|c|c|}
\hline Variable $(==1625)$ & $\mathrm{M}$ & $\mathrm{SD}$ & 1 & 2 & 3 & 4 & 5 & 6 & 7 & 8 & 9 & 10 & 11 & 12 \\
\hline 1 Cohesion & 65.2 & 7.9 & 3 & 2 & 2 & 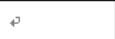 & 2 & 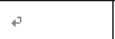 & 3 & 3 & 2 & 3 & 3 & 3 \\
\hline 2Adaptation & 47.3 & 7.7 & $0.755 * *_{0}$ & 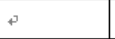 & 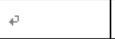 & 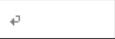 & 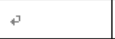 & 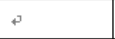 & 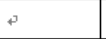 & 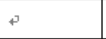 & 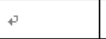 & 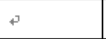 & 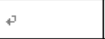 & 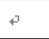 \\
\hline 3Down spirit & 21.7 & 5.4 & $-0.322 * *_{4}$ & $-0.337 * *_{4}$ & 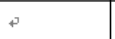 & 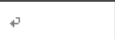 & 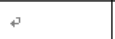 & 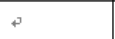 & 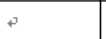 & 3 & 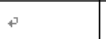 & 3 & 3 & 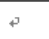 \\
\hline 4Improper conduct & 18.0 & 3.9 & $0.164 * *$ & $-0.413 * *_{p}$ & $0.521 * *$ & 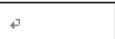 & 3 & 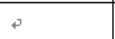 & 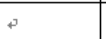 & 3 & 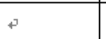 & 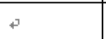 & 3 & 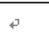 \\
\hline $\begin{array}{l}\text { 5Low sense of } \\
\text { achievement }\end{array}$ & 17.0 & 3.8 & $0.981 * *_{0}$ & $-0.094 *$ & $0.199 * *$ & $0.298 * *$ & 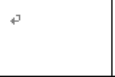 & 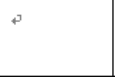 & 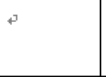 & 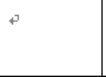 & 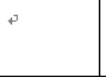 & 3 & 2 & 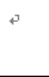 \\
\hline 6Burnout total score & 56.7 & 9.8 & $-0.200 * *$ & $-0.179 * *$ & $0.825 * *$ & $0.811 * *$ & $0.594^{* * *}$ & 2 & 2 & 3 & 2 & 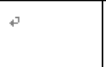 & 2 & 3 \\
\hline 7Target focus & 17.4 & 3.4 & $0.423 * *$ & $0.506 * *$ & $-0.226 * *$ & $-0.289 * *$ & $-0.289 * *$ & $-0.352 * *$ & 2 & 3 & 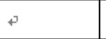 & 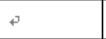 & 3 & 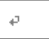 \\
\hline 8Emotion control & 18.9 & 3.9 & $0.482 * *$ & $0.395 * *$ & $0.264 * *$ & $0.151 * *$ & $0.154 * *$ & $0.276^{* *}$ & $0.158 * *$ & 2 & 2 & 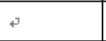 & 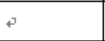 & 3 \\
\hline 9Positive cognition & 14.9 & 3.2 & $0.106 * *$ & $0.454 * *$ & $-0.127 * *$ & $-0.127 * *$ & $-0.207 * *$ & $-0.194 * *$ & $0.544 * *$ & $0.091 *$ & 2 & 3 & 3 & 3 \\
\hline 10Family support & 21.4 & 3.8 & $0.436 * *_{*}$ & $0.531 * *$ & $-0.181 * *$ & $-0.127 * *$ & $-0.152 * *$ & $-0.216 * *$ & $0.363 * *$ & $0.133 * *$ & $0.434 * *$ & 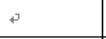 & P & 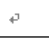 \\
\hline 11Interpersonal help & 19.8 & 4.3 & $0.089 *$ & $0.097 *$ & $-0.206 * *$ & $-0.163 * *$ & $-0.129 * *$ & $-0.237 * *$ & $0.262 * *_{0}$ & $0.271 * *$ & $0.261 * *$ & $0.334 * *$ & 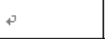 & 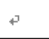 \\
\hline
\end{tabular}

Note: $* P<0.05, \quad * * P<0.01$

\subsection{Mediating Effect Verification}

3.3.1 Verification of the mediating effect of psychological resilience between family cohesion and learning burnout

The stratified regression analysis method is adopted to verify the mediating effect of the total score of juvenile psychological resilience scales between family cohesion and the total score of undergraduates' learning burnout scales. The first step is to use family cohesion as the independent variable and learning burnout as the dependent variable and carry out linear regression analysis. The results give a standardized regression coefficient of $\mathrm{c}=-0.210(\mathrm{P}<0.001)$, which indicates that family cohesion has a remarkable predictive effect on learning burnout; the second step is to use family cohesion as the independent variable and psychological resilience as the dependent variable and carry out linear regression analysis. The results give a standardized regression coefficient of $\mathrm{a}=0.312(\mathrm{P}<0.001)$, which indicates that family cohesion has a remarkable predictive effect on psychological resilience; the third step is to use family cohesion and psychological resilience as independent variables and learning burnout as the dependent variable and carry out linear regression analysis. The results indicate that the standardized regression coefficients of family cohesion and 
psychological resilience to learning burnout are $b=-0.365(P<0.01), c^{\prime}=-0.097(P<0.001)$ respectively. Please refer to table 2 for the results.

Table 2 Verification of the mediating effect of psychological resilience between family cohesion and learning burnout

\begin{tabular}{llccc}
\hline Step & Formula & Result & $t$ & $P$ \\
\hline First & $\mathrm{Y}=\mathrm{cX}+\mathrm{e}$ & $\mathrm{c}=-0.210$ & -8.668 & $<0.001$ \\
Second & $\mathrm{M}=\mathrm{aX}+\mathrm{e}$ & $\mathrm{a}=0.321$ & 13.223 & $<0.001$ \\
Third & $\mathrm{Y}=\mathrm{c}^{\prime} \mathrm{X}+\mathrm{bM}+\mathrm{e}$ & $\mathrm{b}=-0.365$ & -15.282 & $<0.001$ \\
& & $\mathrm{c}=-0.097$ & -4.041 & $<0.001$ \\
\hline
\end{tabular}

3.3.2 Verification of the mediating effect of psychological resilience between family adaptation and learning burnout

This verification takes family adaptation as the independent variable and learning burnout as the dependent variable, then implements the same steps to verify the mediating effect of psychological resilience. Herein the details are omitted, please refer to table 3 for the results.

Table 3 Verification of the mediating effect of psychological resilience between family adaptation and learning burnout

\begin{tabular}{llccc}
\hline \multicolumn{1}{c}{ Step } & Formula & Result & $t$ & $P$ \\
\hline First & $\mathrm{Y}=\mathrm{cX}+\mathrm{e}$ & $\mathrm{c}=-0.213$ & -8.799 & $<0.001$ \\
Second & $\mathrm{M}=\mathrm{aX}+\mathrm{e}$ & $\mathrm{a}=0.379$ & 16.486 & $<0.001$ \\
Third & $\mathrm{Y}=\mathrm{c}^{\prime} \mathrm{X}+\mathrm{bM}+\mathrm{e}$ & $\mathrm{b}=-0.367$ & -14.931 & $<0.001$ \\
& & $\mathrm{c}^{\prime}=-0.074$ & $-\mathbf{3 . 0 2 8}$ & $<\mathbf{0 . 0 0 1}$
\end{tabular}

From 3.3.1 and 3.3.2, we can infer that the mediating effect of psychological resilience is remarkable. In addition, in the third step verification, the standardized regression coefficients of family cohesion and adaptation to learning burnout are of remarkable significance $(\mathrm{P}<0.001)$, so it is a partial mediating effect. The ratio of mediating effect to the total effect is $(-0.365) \times 0.321 /-0.21=0.558$ (family cohesion as the independent variable) and $(-0.367) \times 0.379 /-0.213=0.653$ (family adaptation as the independent variable) respectively, that is to say, the mediating effect explains the dependent variable's variance deviation of $55.8 \%$ and $65.3 \%$.

\section{Discussion}

The total score of learning burnout scales falls in the medium range. Within 3 dimensions, improper conduct gets the highest mean score while low sense of achievement has the lowest, which is consistent with that of former researches ${ }^{\text {[29-31]. }}$ The results indicate that medium learning burnout commonly exist in medical students, mainly reflected by improper learning behavior(such as, truancy, inattention, dilatory learning and so on).

The results of this research also indicate that the family type of most medical students belongs to "regular-intimate(balanced type)", which means the family relation of most medical students is harmonious and their families can make proper treatment and changes internally when facing problems.

Psychological resilience exerts a partial mediating effect both in the influence of family cohesion on learning burnout and in the influence of family adaptation on learning burnout, which indicates that family cohesion and adaptation can not only directly influence learning burnout, but also influence it via psychological resilience. This conclusion is consistent with that of this research due to the following two reasons: one is the cultivation of coping ability, families with high cohesion and adaptation can provide learning guidance and help for their children, making them form correct learning outlook, find scientific learning methods, better adapt to learning environment and overcome learning difficulties, thereby reducing learning burnout ${ }^{[32]}$; the other is emotional support, families with high cohesion and adaptation can also establish excellent family atmosphere and 
parent-child relationship, render children with care and support, encourage them to strive to find problem-solving methods, prompt them to form persistent individuality, improve their psychological resilience ${ }^{[33-34]}$, make them better cope with learning difficulties and restore better and rapidly even facing frustration.

\section{References}

[1] Pines A, Aronson E, burnout: from tedium to personal growth, Free press, New York, 1981.

[2] Lian Rong, Yang Lixian, Wu Lanhua, The Relationship between Undergraduates' Learning Commitment and Learning Burnout and its Scale Formulation[J], Acta Psychologica Sinica, 2005,37(5):632-636.

[3] Seo, J.-H., Kim, H.-J., Kim, B.-J., Lee, S.-J., \& Bae, H.-O. (2015). Educational and Relational Stressors Associated with Burnout in Korean Medical Students. Psychiatry Investigation, 12, 451-458.

[4] Cecil,J., McHale, C., Hart, J., \& Laidlaw, A. Behaviour and Burnout in Medical Students. Medical Education Online, 2014, 19, Article No. 25209. https://doi.org/10.3402/meo.v19.25209

[5] Li Jun, Yin Liqin, Liu Xiaoming(2014). The Influence of Core Self-evaluation on the Learning Burnout of College Students Majoring in Sports: the Mediating Effect of Coping Styles. Sports Technology, 36(3):94-96.

[6] Lv Sixin, Li Lixia, Ke Binbin, Liang Yingyi, Mai Rulan(2014). The Regression Analysis about Learning Burnout of Undergraduates in a Guangzhou College and its Influential Factors. Chinese Journal of School Health, 35(1):120-122.

[7] Dyrbye, L. N., Massie, F. S., Eacker, A., Harper, W., Power, D., Durning, S. J., Thomas, M. R., Moutier, C., Satele, D., Sloan, J., \& Shanafelt, T. D. Relationship between Burnout and Professional Conduct and Attitudes among US Medical Students. JAMA, 2010,304,1173-1180.

[8] Dyrbye, L. N., Harper, W., Moutier, C., Durning, S. J., Power, D. V., Massie, F. S., Eacker, A., Thomas, M. R., Satele, D., Sloan, J. A., \& Shanafelt, T. D. A Multi-Institutional Study Exploring the Impact of Positive Mental Health on Medical Students' Professionalism in an Era of High Burnout. Academic Medicine, 2012, 87, 1024-1031.

[9] Zeng Xiaoying, Re-exploration about the Relationship between Undergraduates' Self-efficacy, Self-esteem and Learning Burnout, Journal of Chengdu Normal Institute. 2014,30(12),90-94.

[10]Qian Kangjie, Yin Keli, Zhang Lirong. The Predictive Effect of Learning Burnout on Undergraduates’ Positive and Negative Psychological Status. Chinese Mental Health Journal. 2015,29(3):236-238.

[11]He Xiangyi. The Relationship between the Learning Burnout and Academic Performance of Students in Local Normal Universities. Modern Education Management, 2011,31(1),72-74.

[12]Zhou Jianming. Research about the Relationship between Knowledge Learning Burnout and Entrepreneurial Vigilance and Performance. Journal of Hubei Economic Institute(Column of Humanistic and Social Sciences). 2014, 11(8):174-178.

[13]Richardson, G. E.. The Meta Theory of Resilience and Resiliency. Journal of Clinical Psychology,2002, 58,307-321.

[14]Haydock, D., Mannix, J., \& Gidman, J. CPTs’ Perceptions of Their Role Satisfaction and Levels of Professional Burnout. Community Practitioner, 2011.84, 19-23.

[15]Ronen, S., \& Mikulincer, M. Predicting Employees' Satisfaction and Burnout from Managers' Attachment and Care-giving Orientations. European Journal of Work and Organizational Psychology, 2012,21, 828-849.

[16]Ma Xiting, Li Ran, Deng Lifang. Research about the Relationship between Undergraduates‘ Family Cohesion and Adaptation and their Personality Development. Chinese 
Journal of Health Psychology. 2009,17(9):1073-1075.

[17]Hou Yezhi, Xiang Yutao, Li Wenyong, Gao Liang, Chen Guolan, Wong Yongzhen. Research about the Correlation between Family Cohesion and Adaptation and Social Functions of 70 Schizophrenics from Beijing Communities. Chinese Journal of Clinical Rehabilitation. 2003,7(21):2946-2947.

[18]Wang Lei, Hong Dailing. Investigation and Research about Family Cohesion and Adaptation of Leukemia Children in Catabasis. Journal of Nursing Science. 2007,22(23):25-27.

[19]Fei Lipeng and etc., Preliminary Evaluation about "Family Cohesion and Adaptation Scale" and "Family Environment Scale", Chinese Journal of Mental Health, 1991,5(5):198-202.

[20]Hou Yongmei, Hu Peicheng, Wang Yiyang. The Relationship between Undergraduates' Psychological Resilience and Learning Burnout: the Mediating Effect of Coping Styles. Advances in Psychology, 2017,7(7).

[21]Wang Pengjun, Zhang Shichao. The Relationship between Middle School Students' Life Incidents, Psychological Resilience and Learning Burnout. Science of Social Psychology. 2011,26(8):95-98.

[22]Sun Jihong, Liu Ran, Lv Honghong. The Influence of Family Cohesion on Psychological Resilience of Enterostomy Children. Journal of Clinical and Pathology. 2017,37(2):372-378.

[23][23]Tan Huayu. The Correlation between Family Environment, Occupational Self-efficacy and Learning Burnout---Based on the Empirical Research in 5 Colleges of Guangdong Province. Education Research Monthly 2013,29(6):59-63.

[24]He Jianxin, Gong Yuehua, Wu Jie. Social Influence Factors of Learning Burnout of Higher Vocational Students and Countermeasure Research[J]. Career Horizon, 2010,6(7):159-160.

[25]Fei Lipeng. Family Cohesion and Adaptation Scale(FACES II - CV)[J]. Wang Xiangdong, Wang Xilin, Ma Hong. Manual of Mental Health Evaluation Scale. Chinese Mental Health Journal, 1999(supplementary issue):142-149.

[26] Hu Yueqin, Gan Yiqun. Formulation of Juvenile Psychology Resilience Scale and Efficacy Verification. Journal of Psychology, 2008,40(8):902-912.

[27] Olson D, Russel C, Spenkle D. Cirupolar Model of Marital and Family System VI: Theoretical Update [J]. Family Process, 1983,22: 69-83.

[28]Wen Zhonglin, Hou Jietai, Zhang Lei. Comparison and Application of the Regulating Effect and Medicating Effect. Journal of Psychology. 2005.28(2),268-274.

[29] Shu HL, Yun CH. Investigation the relationships between loneliness and learning burnout [J]. Act Learn High Edu, 2012， 13(3):231-243.

[30]Xiao Jing, Wang Fei, Ge Hua, Li Fuye, Lian Yulong. The Relationship between Medical Students' Learning Burnout and Emotional Intelligence. Chinese Journal of School Health. 2013,34(12):1442-1446.

[31]Liang Peiyu, Yin Xiran, Han Jiming, Chen Zhuangding, Li Bin, Liu Shou. Correlation Research about Medical Students' Learning Burnout and Anxiety Status Quo and their Correlative Factors. Henan Journal of Preventive Medicine. 2017,28(6):401-405.

[32] SHEK DANIEL T L. Family function and psychological w ell- being, school adjustment, and problem behavior in Chinese adolescents with and wi th out economic disadvantage[J]. Genetic Psychol, 2006,163(4): 497-500.

[33] YANG Qing, YI Lilan, SONG Wei, et al. The relationship between loneliness and family cohesion and school belonging of left behind children in rural areas [J]. Chinese Journal of mental Health, 2016,30(3): 197-201.

[34]Li Shasha, Li Sasa, Dong Xiaocui. The Influence of Negative Emotion Regulation Ability and Tenacious Personality of Nursing Undergraduates on their Emotional Resilience[J]. Chinese Journal of Practical Nursing, 2014,30(2):19-22. 